\title{
Amelioration of soils contaminated with radionuclides: Exploiting biodiversity to minimise or maximise soil to plant transfer
}

\author{
N. Willey \\ Centre for Research in Plant Science, Faculty of Applied Sciences, University of the West of \\ England, Coldharbour Lane, Frenchay, Bristol BS16 1QY, UK, \\ e-mail: Neil.Willey@uwe.ac.uk
}

\begin{abstract}
There is much current interest in managing and manipulating radionuclide transfer from soils to plants, either for 'safe' crops or for phytoextraction of radionuclide contaminated soils. Potentially there is biodiversity in plant uptake that might aid these efforts. We have established the effects of phylogeny (evolutionary history) on the uptake of numerous heavy metal and nutrient elements. Here, using the same Residual Maximum Likelihood techniques, we report phylogenetic effects on radionuclide uptake by plants derived from databases of several hundred species of plants we have collated over recent years. There are differences of at least two orders of magnitude in the concentration to which plants take up isotopes of $\mathrm{Cs}, \mathrm{Sr}, \mathrm{Cl}, \mathrm{Ru}, \mathrm{S}$, and I. When analysed using hierarchical Analysis of Variance coded using a recent molecular phylogeny of flowering plants it is clear that for no radionuclide is all the variation inter-specific. Rather, for all radionuclides some of the inter-taxa variation is associated with higher taxonomic levels. In general, therefore, groups of plants with high and low uptake of radionuclides, and that might be useful for managing or manipulating radionuclide uptake, can be delineated. Here we discuss some of these groups and how they might be useful.
\end{abstract}

\section{INTRODUCTION}

Managing the transfer of radionuclides from soils to plants, and thence to food chains, is an important post-accident challenge for radioecologists. There is increasing potential for manipulating the soil to plant transfer of radionuclides to help meet this challenge. Here we show that there is significant biodiversity in soil to plant transfer of radionuclides that might be exploited in both managing and manipulating radionuclide transfer. We suggest a framework based on recent phylogenies of flowering plants that might be used to exploit the biodiversity in soil to plant transfer of radionuclides and aid the amelioration of soils contaminated with radionuclides post-accident.

The evolutionary history (phylogeny) of an organism constrains many of its traits but until recently the understanding of the phylogenies of organisms was insufficient to enable rigorous quantification of these constraints. In the last decade major advances in computer algorithms, computing power and the generation of gene sequence information have coalesced to provide phylogenies of unprecedented detail for many groups of organisms, and indeed the living world as a whole (1). The Angiosperm Phylogeny Group (APG) has provided progressively more detailed phylogenies for flowering plants (see 2 and fig 1.) and plant biologists have used these to quantify the effects of phylogenetic position on variation in a number of traits in plants, e.g. mycorrhization (3) and $\mathrm{N}$-fixing symbioses (4). The new phylogenies for flowering plants differ in some important respects from the previous understanding of the evolutionary history of flowering plants and have been instrumental in clarifying differences in the various traits across flowering plants. 


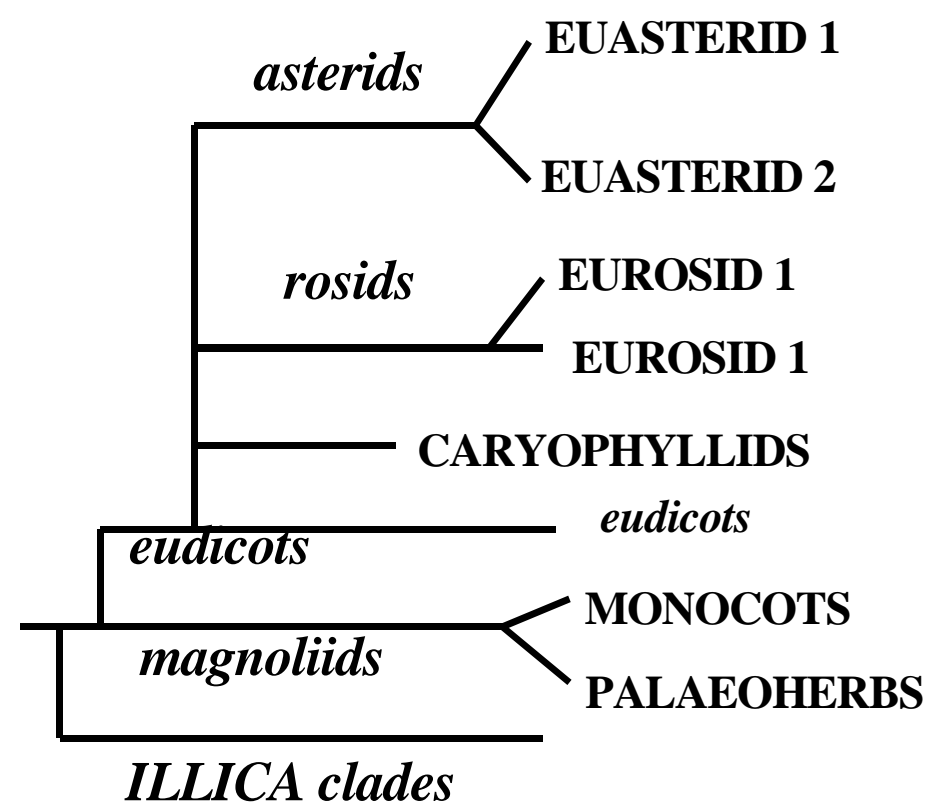

Figure 1. A generalised phylogeny of flowering plants based on APG II (2004) (ref 2).

We hypothesised that plant uptake of mineral elements might be constrained by phylogeny and that this might be useful for identifying plants with high and low uptake of mineral elements. We first showed, using 137 species, that radiocaesium uptake by a plant species is affected by the position of the species on the flowering plant phylogeny (5). The analytical techniques were then used to investigate phylogenetic affects for a suite of heavy metals including $\mathrm{Zn}, \mathrm{Cd}, \mathrm{Cu}, \mathrm{Ni}, \mathrm{Pb}$ and $\mathrm{Co}(6)$. Subsequent analyses have focused on quantifying the phylogenetic effects on the uptake of Ca (7) and a suite of nutrient ions (8). It now seems that different branches (clades) of the phylogeny of flowering plants have some mineralogical characteristics that derive, ultimately, from their uptake of ions from the soil. We have now generated and collated soil to plant transfer data for numerous species for isotopes of $\mathrm{Cs}, \mathrm{Co}, \mathrm{S}, \mathrm{I}, \mathrm{Sr}, \mathrm{Cl}$ and $\mathrm{Ru}$ and mapped it onto a new phylogeny of the flowering plants. Here I present, for the first time, an overview of the phylogenetic effects on plant uptake of radionuclides and compare it to those for other elements. I show that there are significant phylogenetic effects in soil to plant transfer factors for radioisotopes and discuss the ways in which this might aid the identification of plants with low and high uptake, and hence the management and manipulation of soil to plant transfer. This might be particularly useful in developing safe crops for radionuclide contaminated soils.

\section{METHODS}

In order to map variation in a trait such as soil to plant transfer onto a phylogeny, large inter-taxa comparisons are necessary. There are many datasets in the radioecological literature that compare transfer factors for radionuclides in different species of plants. We developed a statistical method based on REML analysis for collating these datasets together into databases for each radionuclide. If datasets have species in common these can be used to normalise values across datasets to form the databases. Very often, however, datasets do not have species in common, and even if they do they produce databases that are phylogenetically unbalanced, e.g. they might contain values for cereal 
crops and not much else. To overcome this problem we generate datasets of our own which include both species that can link other datasets together plus species that increase the phylogenetic spread of data. Clearly, the resultant databases then provide an estimate of relative transfer factors across datasets that are derived from a variety of conditions. The different conditions under which the data are derived is in many ways an advantage as it provides an estimate of the averaged behaviour across different conditions. It is tempting to think that it would be better to generate, experimentally, one large inter-comparison of species under one set of conditions for phylogenetic analysis. This is not necessarily the case because different plants behave differently under different conditions and an average of their behaviours under a variety of conditions is in many ways a preferable estimate of the effect of phylogeny.

We select for inclusion, and generate experimentally, radionuclide concentrations in green shoots in the absence of any foliar deposition. Data in these sets must have radionuclide concentrations measured simultaneously in three or more taxa under the same environmental conditions. We then use these datasets, either from the literature or experiments, as blocks in a Residual Maximum Likelihood Analysis and then a hierarchical Analysis of Variance based on the phylogeny of flowering plants, to estimate relative transfer factors for species and all other levels of the taxonomic hierarchy. For Ca the proportion of all taxa that need to be sampled at the different taxonomic levels for statistical significance has been determined (7), and we assume that these proportions apply to other minerals. The method has now been widely published $(5,6,7,8)$ and, although not without limitations, generates the most detailed quantifications of the effects of phylogeny on elemental uptake by plants yet attempted.

\section{RESULTS}

By normalising data in the literature with data we have generated experimentally, we have constructed databases from which REML and ANOVA estimate relative concentrations of Cs in 237 taxa, of Sr in 108 taxa, of $\mathrm{Cl}$ in 106 taxa, of Ru in 114 taxa, of S in 121 taxa and of I in 60 taxa. These are the databases with the widest taxonomic spread yet produced for radionuclides and approach the taxonomic spread of databases for heavy metals (5).

Table 1. The \% Sum of Squares at each level of the taxonomic heirarchy for six radionuclides $(\mathrm{NS}=$ not a significant contributor to overall variation at $<0.05$ ) using heirarchical ANOVA based on Angiosperm Phylogeny Group phylogeny.

\begin{tabular}{|c|c|c|c|c|c|c|}
\hline & Cs & Cl & $\mathbf{R u}$ & S & 1 & \\
\hline Number of Species & 237 & 103 & 106 & 114 & 121 & 60 \\
\hline Class & 4.2 & 4 & 2.6 & 4.2 & 1.0 & ${ }^{\mathrm{NS}} 0.21$ \\
\hline Subclass & 6.3 & 1.5 & ${ }^{\mathrm{NS}} 0.22$ & 8.9 & 5.0 & ${ }^{\mathrm{NS}} 0.79$ \\
\hline Group & 22.1 & 3.17 & ${ }^{\mathrm{NS}} 0.43$ & ${ }^{\mathrm{NS}} 0.22$ & 5.1 & 4.2 \\
\hline Superorder & $\mathrm{NS}_{2.3}$ & ${ }^{\mathrm{NS}} 1.55$ & 4.8 & 2.0 & 3.0 & ${ }^{\mathrm{NS}} 0.78$ \\
\hline Order & 7.4 & 11.7 & 37.6 & 23.8 & 21.8 & 13.5 \\
\hline Family & 6.2 & 12.9 & 6.6 & 11.1 & 20.5 & 1.4 \\
\hline Genus & 35.2 & 55.3 & 32.5 & 41.1 & 29.8 & 75.3 \\
\hline Species & 16.3 & 8.7 & 15.3 & 8.7 & 13.8 & 3.7 \\
\hline
\end{tabular}


In order to understand the usefulness of the results of the phylogenetic analyses it is necessary to understand the strengths and weaknesses of the method. Although the databases generate a single relative value for concentration in each taxon, these are frequently based on many original values in the datasets. For example, wheat and barley appear in many of the datasets and hence the estimates of their relative concentrations (which are quite similar) are likely to be more reliable than those of taxa that appear a single time. It is probably desirable to have many independent replicate values for each taxa, and for this reason our experimental data are means of five replicates. However, statistically it is at least as important to have as wide a taxonomic spread as possible as it is to have multiple values for a single taxon. Clearly, if there are differences between taxa in the uptake of radionuclides when they are grown on a single contaminated substrate, the rank order of concentration might change with substrate, i.e. there might be a statistical interaction between environmental conditions and relative radionuclide concentrations. In our analysis we treat environmental conditions as blocks - effectively removing their relative effects from the data to reveal the effects of taxon. We take no account of possible interactions because most of the blocks of environmental conditions are not replicated.

Despite the limitations outlined above, when databases of relative raionuclide concentrations are analysed using hierarchical ANOVA with factors nested according to the APG phylogeny there are numerous statistically significant effects. If some of the limitations outlined above could be overcome then effects would probably be more, rather than less, significant. Table 1 summarises whereabouts on the taxonomic hierarchy the \% of the total sum of squares attributable to phylogeny reside. In general, between about $30 \%$ to $50 \%$ of all variance in radionuclide concentration is attributable to phylogeny. For ${ }^{137} \mathrm{Cs}$, ${ }^{35} \mathrm{~S}$ and ${ }^{109} \mathrm{Cd}(9)$ e have investigated the effects of intervarietal differences on the analysis (see Fig 1). Within a species there are clearly significant differences in uptake between varieties (see Fig 1). There are data on inter-varietal differences in radionuclide uptake for very few species and the phylogenetic relationships between many varieties is unclear. At least a proportion of the variance not as yet ascribed to phylogeny might, therefore, be so if such analyses of varieties was possible. Finally, for all radionuclides, the relative concentrations indicate that there are differences of at least 100-fold, often more, between concentrations in different taxa.

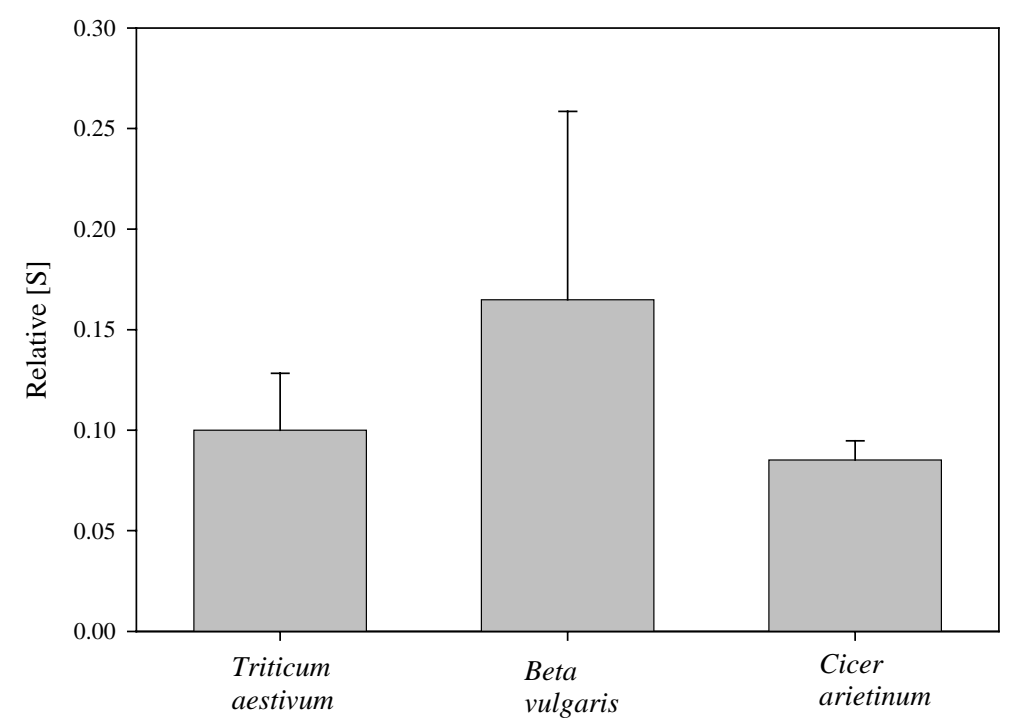

Figure 2. Mean relative and standard error of $S$ concentrations in 12 varieties of $T$. aesitvum (wheat), 16 varieties of $B$. vulgaris (beet) and 8 varieties of $C$. arietinum (Chickpea). 
For Cs, all the taxonomic units with the exception of superorder can explain a statistically significant proportion of the variance in the database of relative concentration values. In particular the group, and in particular the Caryophyllidae, is associated with significant proportion of the variance. The analysis in Table 1, which includes many more data than previously published (5), including many generated for Chinese taxa by Dr Shirong Tang of Zheijang University, emphasises the importance of the Caryophyllidae but the detail also indicates that the Asteracae include a number of taxa with high Cs uptake. The Sr results in table 1 are in accord with those for Ca (7) with genera in the Cucurbitales, Malvales and Lamiales having notably high uptake. Similarly, $\mathrm{Cl}$ has high uptake in the Cucurbitales, particularly in certain genera. Overall, for $\mathrm{Cs}, \mathrm{Sr}, \mathrm{Cl}$ and $\mathrm{Ru}$, taxa in classes that include the 'primitive' monocotyledonous plants have statistically significantly lower uptake than those on the Rosid, Asterid and Caryophyllid clades. This confirms many general observations that cereal crops have lower uptake, on average, of these radioisotopes than many vegetable crops for example.

For both $\mathrm{S}$ and I there are particular groups that have low and high uptake but they can be located on either 'primitive' or 'advanced' clades. For S in our analyses, both the Lilliales (Monocots) and Brassicales (Rosids) have high uptake. For Iodine genera in both the Poales (Grasses and allies) and Fabales (Legumes and allies) have high uptake.

\section{DISCUSSION}

If each plant species took up a radionuclide in absolutely its own way then no variance would be attributable to other levels of the phylogeny and $100 \%$ of the sum of squares would be in the species category in table 1 . This is clearly not the case for radionuclides, nor is it for various stable elements, and there are clearly phylogenetic effects on plant uptake of elements. It is pertinent, however, to ask whether this biodiversity might be useful for amelioration of soils contaminated with radionuclides. In general, the analyses reported not only begin to quantify the biodiversity available for managing soil to plant transfer of radionuclides, they also suggest that a phylogenetic framework might provide a means for exploiting it.

There is current interest in 'safe' crops that might be grown on radionuclide contaminated soils with minimal uptake of radionuclide. We have suggested that phylogeny might be a useful background to research for minimising radiocaesium uptake by plants (10) as there are clearly identifiable groups of plants that have low uptake of radionuclides. For example, I predict that the plant with the lowest Cs uptake will be a variety of a plant in the Poaceae or their allies. It might take some time to find it as the number of such plants is huge, but phylogeny at least provides some idea of where it might be. Because it's time consuming to search through the Poaceae for a low uptake plant the generation of 'safe' crops is focusing of breeding crops with well-known genetics or utilising Arabidopsis resources. If such studies identify genotypes associated with low Cs uptake they might provide marker genes for searches within the Poaceae. There is also current research into Cs uptake mechanisms in plants, often with a view to genetically manipulating Cs uptake. When the genes associated with uptake mechanisms for Cs have been identified (11), or when utilising Quantitative Trail Loci that have already been described in Arabidopsis (12), the biodiversity 'landscape' described through phylogeny could provide a useful conduit to application in the field. This might be for safe crops or for those with enhanced uptake of radioceasium. In general, plant uptake of radiocaesium from many soils is not fast enough to make phytoextraction a viable decontamination option. However, physiological and genetic studies that access biodiversity in Fe or As uptake, elements that are very unavailable to plants in many soils, have made significant recent advances and it seems possible that plants with enhanced Cs uptake and mobilisation capacities might be engineered (13). For all other radionuclides the physiological and genetic understanding of uptake is less advanced than for Cs, ensuring that the biodiversity 'landscapes' we are describing are amongst the few frameworks getting a handle on exploitable biodiversity. 
The databases of relative radionuclide concentrations we have collated also hold other, perhaps more subtle, insights that might be useful for post-accident decontamination of soils. For example, most of the databases have relative radionuclide concentrations that are lognormally distributed. This has previously been reported but is infrequently taken account of in models of soil to plant transfer. Further, the fact that not all the variance resides at the species level but at higher taxonomic levels means that species are not independent samples for radionuclide concentration. Many of the statistics used in radioecology assume that they are. This might be reducing the statistical power of analyses used in responding to radionuclide contaminated soils. Finally, there seems to be no reason why the species should be a category of particular radioecological focus. For many radionuclides there is more variance associated with other categories.

To conclude, there is great biodiversity in plant uptake of radionuclides which is partly explained by flowering plant phylogeny and is therefore, in general terms at least, predictable. This might be a useful framework for advanced studies focused on managing and manipulating radionuclide transfer from soils to plants in post-accident scenarios.

\section{Acknowledgements}

I would like to thank the Food Standards Agency and the Leverhulme Trust who both helped to fund this research.

\section{References}

[1] Science Special Issue, $13^{\text {th }}$ June, v 300, 1691-1709.

[2] Angiosperm Phylogeny Group 2003. Bot. J. Linn. Soc. 141, 399-436. (2003).

[3] A.H. Fitter, B. Moyerson. Philos. Trans. Royal Soc. B 351, 1376-1375. (1996).

[4] Doyle J. Trends Plant Sci. 3, 473-478. (1999).

[5] M.R. Broadley, N.J. Willey, A. Meade. Environ. Pollut. 106, 341-349. (1999).

[6] M.R. Broadley, Willey, N.J., Wilkins, J.C., Baker, A.J.M., Mead, A., White, P.J., New Phytol. 152, 9-27. (2001).

[7] M.R. Broadley, Bowen, H.C., Cotterill, H.L., Hammond, J.P., Meacham, M.C., Mead, A., White P.J. J. Exp Bot. 54, 1-16. (2003).

[8] M.R. Broadley, Bowen, H.C., Cotterill, H.L., Hammand, J., P., Meacham, M.C., Mead, A., White, P.J. J. Exp. Bot. 55, 321-336. (2004).

[9] N.J. Willey, R. Bennett. Plant Soil in press (2005).

[10] P.J. White, Swarup, K, Ecobar-Gutiérrez, A.J., Bowen, H.C., Willey, N.J., Broadley, M.R., Plant Soil, 249, 177-186. (2003).

[11] M.R. Broadley, Escobar-Gutiérrez, A.J., Bowen, H.C., Willey, N.J., White, P.J., J. Exp. Bot. 52, 839-844. (2001).

[12] K.A. Payne, K.A., Bowen, H.C., Hammond, J.P., Hampton, C., Lynn, J.R., Mead, A., Swarup, K., Bennett, M.J., White, P.J., Broadley, M.R. New Phytol. 162, 535-548. (2004).

[13] N.J. Willey (Ed) Phytoremediation: Methods and Reviews. Humana Press, NJ, USA. (2005). 